\title{
Editorial to the Special Issue on Psychological and Biological Time: The Role of Personality
}

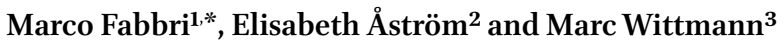 \\ ${ }^{1}$ Department of Psychology, University of Campania Luigi Vanvitelli, Caserta, Italy \\ ${ }^{2}$ Department of Psychology, Umeå University, Umeå, Sweden \\ ${ }^{3}$ Institute for Frontier Areas of Psychology and Mental Health, Freiburg, Germany
}

Time offers a dimension for all our activities, is essential for perception and defines our behaviour. Time is part of our life and defines us through our lifespan. Several time dimensions have therefore been defined:

(1) Time perception (TP) has a fundamental impact on individuals' optimal functioning, and it can be characterized as the matching or mismatching between objective and subjective time. Two main concepts constitute our experience of time: succession (i.e., identification of event temporal order) and duration (i.e., identification of event persistence over time). However, different personality and psychiatric disorders, including developmental issues, such as depression, anxiety and impulsivity, can impact the perception of time (Lake, 2016; Wittmann \& Paulus, 2008).

(2) Experience is cognitively parsed or tagged into separable time zones, as the time perspective or time orientations (TO) which play a role in the construction of human experience into past, present, and future temporal frames (Zimbardo \& Boyd, 1999). This cognitive construction provides order, coherence, and meaning for personal and social experience (Carelli e al., 2011; Wittmann \& Sircova, 2018). Thereby, it is important to reach a healthy balance between the time orientations as this balance can be considered the ability to learn from the past, to adapt in the present, and to engage in goaloriented behavior in the future. Personality traits can be related to different

\footnotetext{
* To whom correspondence should be addressed. E-mail: marco.fabbri@unicampania.it
} 
time orientations (Åström et al., 2019) as well as to a deviation from the balanced time perspective.

(3) Time awareness (TA) is defined as the subjective impression of time passing quickly or slowly. This is also associated with personality dimensions: for example, impulsive or boredom-prone individuals complain that time passes too slowly and, therefore, react prematurely (Jokic, Zakay \& Wittmann, 2018).

(4) Finally, circadian typology indicates individual circadian rhythms (CR) from a biological approach. Morningness/eveningness preference has been widely associated with personality, suggesting, for example, that morning-types are more conscientious, whereas evening-types are more related to extraversion or neuroticism (Tonetti et al., 2009, 2016) with an impact on social behavior (Fabbri et al., 2007).

Research on the connections between the different time dimensions is scarce and unsystematic, especially those indicating the role of personality in individual 'temporal' differences. This Special Issue on 'Psychological and Biological Time: The Role of Personality' aims to fill the gap in the literature, showing how different time dimensions are interrelated and connected to individual differences and personality development.

The five articles in this Special Issue can be sorted into two categories, namely (1) the question of influences of individual differences (personality) on the perception of time (three articles) and (2) the effects of trait-like time perspectives in individuals with problematic drinking behavior and in patients with borderline personality disorders (two articles).

Concerning influences of personality on time perception, Roy, Fortin-Guichard, Tétreault, Laflamme \& Grondin (this issue) investigated the relationship between temporal personality of university students in psychology, their personality traits and their preferences for each of the four main theoretical orientations (i.e., psychodynamic-analytic, cognitive-behavioral, existential-humanistic and systemic-interactional orientations), considering that in addition to personality traits, several psychotherapeutic techniques aim to modify the client's attitude and beliefs in relation to past, present and/or future. The results showed that students with a preference for the existential-humanistic and systemic-interactional orientations were associated with weaker tendencies for proximity of the results, consciousness of time on vacation and planning at home. Moreover, students with a preference for the cognitive-behavioural orientation were associated with stronger planning skills, time awareness and a propensity for conscientiousness. This study is interesting because it presents how the temporal framework of each theoretical orientation, whether in terms of therapeutic modalities or clinical conceptualization, challenges individuals in their way of managing their task and reacting to time. 
Bisson \& Grondin (this issue), in a further attempt to assess the relationship between personality and time perception, had subjects undertake an internet surfing task (an activity where one easily loses track of time) and assess prospective and retrospective duration judgments with a within-subject design. This is likely the first of its kind to compare these two perspectives within one study within the same group of participants. First of all, prospective estimates on average appeared to be significantly longer than retrospective duration estimates (in 58\% of subjects this was the case) and they were more variable. Regarding personality influences, individuals who relatively overestimated duration in the prospective and retrospective paradigm had lower leisure-time awareness and showed less time use and planning at home as well as at work. This is an interesting finding as it relates a specific task (time orientation while surfing the internet in a laboratory situation) with time orientation as personality in everyday tasks.

Koswara, Widyanti \& Park (this issue) aimed to observe the role of personality in perceived time load (defined as the extent to which time pressure was felt due to the pace at which the tasks or task elements occurred) in participants with different HEXACO personalities. All voluntary participants performed a visual search task in conditions with different levels (simple, one-counter and three-counter conditions) of difficulty, representing different time pressures; at the end of each condition the perceived time load was assessed. With comparable performance, the results showed that there was a strong tendency towards significance of personality in the perceived time load in general, and extravert people tended to rate perceived time load higher than other personality types in the difficult tasks. These findings can be explained referring to several models which describe the compromise between temporal and nontemporal information processing with a role played by personality factors.

Concerning changes in time perspective due to problematic behavior or psychopathology, in a larger group of students Loose, Acier \& El-Baalbaki (this issue) investigated the relationship between alcohol use and time personalities such as related to past, present, and future and other temporal as well as non-temporal personality scales. To name just a few findings, a combination of temporal scales, namely the past negative, the present hedonistic as well as the future orientation from the Zimbardo Time Perspective Inventory (ZTPI; Zimbardo \& Boyd, 1999) as well as other personality scales predicted the amount of alcohol use. The past negative and the present hedonistic orientation predict more and the future orientation predicts less alcohol use. The Big-Five subscales either directly (Conscientiousness as correlated with the future dimension) predicted less alcohol use and the effects of the subscales of Openness and Neuroticism on alcohol use were carried by meditators of temporal scales (past negative and present hedonistic).

In the work by Mioni, Wittmann, Prunetti \& Stablum (this issue) patients with borderline personality disorders (BPD) were compared to matched healthy control subjects in the assessment of the time perspective (employing the ZTPI) and the subjective passage of time for present and past time intervals. Strikingly, patients 
with BPD are different in almost all time orientations. They have lower scores in the future and the past-positive dimension, and they have higher scores in the present-hedonistic and past-negative dimensions. Regarding the felt passage of time, patients with BPD feel a general expansion of time at present. The authors discuss their findings in a way that characterizes BPD as instability in affect regulation and impulse control which is associated with an imbalance in individual time orientation and a negatively felt expansion of subjective time in daily life.

\section{References}

Åström, E., Rönnlund, M., Adolfsson, R., \& Carelli, M. G. (2019). Depressive symptoms and time perspective in older adults: associations beyond personality and negative life events. Aging Ment. Health, 23, 1674-1683.

Bisson, N., \& Grondin, S. (2020). A new perspective on the relationships between individual factors and time estimates. Timing Time Percept., 8, 25-54.

Carelli, M. G., Wiberg, B., \& Wiberg, M. (2011). Development and construct validation of the Swedish Zimbardo time perspective inventory. Eur. J. Psychol. Assessment, 27, 220-227.

Fabbri, M., Antonietti, A., Giorgetti, M., Tonetti, L., \& Natale, V. (2007). Circadian typology and style of thinking differences. Learn. Individ. Differ., 17, 175-180.

Jokic, T., Zakay, D., \& Wittmann, M. (2018). Individual differences in self-rated impulsivity modulate the estimation of time in a real waiting situation. Timing Time Percept., 6, 71-89.

Koswara, R., Widyanti, A., \& Park, J. (2020). The role of HEXACO personality in perceived time load. Timing Time Percept., 8, 55-65.

Lake, J. I. (2016). Recent advances in understanding emotion-driven temporal distortions. Curr. Opin. Behav. Sci., 8, 214-219.

Loose, T., Acier, D., \& El-Baalbaki, G. (2020). Personality traits impact alcohol consumption through subjective time. Timing Time Percept., 8, 66-85.

Mioni, G., Wittmann, M., Prunetti, E., \& Stablum, F. (2020). Time perspective and subjective passage of time in patients with borderline personality disorders. Timing Time Percept., 8, 86-101.

Roy, M.-L., Fortin-Guichard, D., Tétreault, E., Laflamme, V., \& Grondin, S. (2020). Temporal personality of psychology students according to their preference for a theoretical orientation. Timing Time Percept., 8, 5-24.

Tonetti, L., De Pascalis, V., Fabbri, M., Martoni, M., Russo, P. M., \& Natale, V. (2016). Circadian typology and the Alternative Five-Factor Model of personality. Int.J. Psychol., 51, 332-339.

Tonetti, L., Fabbri, M., \& Natale, V. (2009). Relationship between circadian typology and big five personality domains. Chronobiol. Int., 26, 337-347.

Wittmann, M., \& Paulus, M. (2008). Decision making, impulsivity and the time perception. Trends Cogn. Sci., 12, 7-12. doi:10.1016/j.tics.2007.10.004.

Wittmann, M., \& Sircova, A. (2018). Dispositional orientation to the present and future and its role in pro-environmental behavior and sustainability. Heliyon, 4, e00882. doi: 10.1016/j.heliyon .2018.e00882.

Zimbardo, P. G., \& Boyd, J. N. (1999). Putting time in perspective: A valid, reliable individualdifferences metric. J. Pers. Soc. Psychol., 77, 1271-1288. 